\title{
Fragment Kinetic Energies and Modes of Fragment Formation
}

T. Odeh, ${ }^{1, *}$ R. Bassini, ${ }^{2}$ M. Begemann-Blaich, ${ }^{1}$ S. Fritz, ${ }^{1}$ S. J. Gaff-Ejakov, ${ }^{3}$ D. Gourio, ${ }^{1}$ C. Groß, ${ }^{1}$ G. Immé,${ }^{4}$ I. Iori, ${ }^{2}$ U. Kleinevoß, ${ }^{1, *}$ G. J. Kunde,,${ }^{3 \dagger}$ W. D. Kunze, ${ }^{1}$ U. Lynen, ${ }^{1}$ V. Maddalena, ${ }^{4}$ M. Mahi, ${ }^{1}$ T. Möhlenkamp, ${ }^{5}$ A. Moroni, ${ }^{2}$ W. F. J. Müller, ${ }^{1}$ C. Nociforo, ${ }^{4}$ B. Ocker, ${ }^{6}$ F. Petruzzelli, ${ }^{2}$ J. Pochodzalla, ${ }^{1,}$ G. Raciti, ${ }^{4}$ G. Riccobene, ${ }^{4}$ F. P. Romano, ${ }^{4}$ A. Saija, ${ }^{4}$ M. Schnittker, ${ }^{1}$ A. Schüttauf,,${ }^{6}{ }^{\S}$ C. Schwarz, ${ }^{1}$ W. Seidel,${ }^{5}$ V. Serfling, ${ }^{1}$ C. Sfienti, ${ }^{4}$ W. Trautmann, ${ }^{1}$ A. Trzcinski, ${ }^{7}$ G. Verde,${ }^{4}$ A. Wörner, ${ }^{1}$ Hongfei Xi, ${ }^{1}$ and B. Zwieglinski ${ }^{7}$

${ }^{1}$ Gesellschaft für Schwerionenforschung, D-64291 Darmstadt, Germany

${ }^{2}$ Istituto di Scienze Fisiche, Università degli Studi di Milano and I.N.F.N., I-20133 Milano, Italy

${ }^{3}$ Department of Physics and Astronomy and National Superconducting Cyclotron Laboratory, Michigan State University, East Lansing, Michigan 48824

${ }^{4}$ Dipartimento di Fisica dell' Università and I.N.F.N., I-95129 Catania, Italy

${ }^{5}$ Forschungszentrum Rossendorf, D-01314 Dresden, Germany

${ }^{6}$ Institut für Kernphysik, Universität Frankfurt, D-60486 Frankfurt, Germany

${ }^{7}$ Soltan Institute for Nuclear Studies, PL-00-681 Warsaw, Hoza 69, Poland

(Received 15 December 1999)

\begin{abstract}
Kinetic energies of light fragments $(A \leq 10)$ from the decay of target spectators in ${ }^{197} \mathrm{Au}+{ }^{197} \mathrm{Au}$ collisions at $1000 \mathrm{MeV}$ per nucleon have been measured with high-resolution telescopes at backward angles. Except for protons and apart from the observed evaporation components, the kinetic-energy spectra exhibit slope temperatures of about $17 \mathrm{MeV}$, independent of the particle species, but not corresponding to the thermal or chemical degrees of freedom at breakup. It is suggested that these slope temperatures may reflect the intrinsic Fermi motion and thus the bulk density of the spectator system at the instant of becoming unstable.
\end{abstract}

PACS numbers: 25.70.Pq, 21.65.+f, 25.70.Mn

Statistical multifragmentation models have been found to be remarkably successful in describing the partition space populated in nuclear reactions leading to multifragment emission [1-3]. This is particularly true for spectator decays following heavy-ion collisions at relativistic bombarding energies. Fragment yields and fragment correlations have been reproduced with high accuracy [4-13], including their dispersions around the mean behavior [14]. The model temperatures are in good agreement with measured isotope temperatures, and the isotopic yields from which these temperatures are derived are well reproduced $[10,13]$.

The mean kinetic energies of the produced particles and fragments, on the other hand, are not universally accounted for if the same set of model parameters is used $[9,10]$. Measured kinetic energies or, equivalently, the slope temperatures obtained from Maxwell-Boltzmann fits to the kinetic energy spectra have been found to considerably exceed the predicted values. This holds even though the effects of Coulomb repulsion and sequential decay cause the calculated slope temperatures to be higher than the equilibrium temperatures in the model description. For the ${ }^{197} \mathrm{Au}+{ }^{197} \mathrm{Au}$ reaction studied in this paper, the measured isotope temperatures and the calculated equilibrium temperatures are 6 to $8 \mathrm{MeV}$ for most of the impact parameter range; the calculated slope temperatures for light particles are about $10 \mathrm{MeV}$ while the measured slope temperatures exceed $15 \mathrm{MeV}$ [10].

The qualitative difference between the chemical or thermal equilibrium temperatures and the kinetic tempera- tures has been observed for a variety of reactions and numerous reasons for it have been presented [15-19]. Preequilibrium or prebreakup emissions are likely explanations for high-energy components in light-particle spectra $[4,10,20,21]$. In some cases, the excess kinetic energies have been ascribed to collective flow even though a characteristic proportionality to the fragment mass is not evident in the data $[11,22,23]$. This situation caused other authors to more generally question the applicability of statistical descriptions for spectator fragmentation [24]. An explanation in terms of the Goldhaber model [25] that is favored here has been suggested long ago $[15,26]$ but, so far, has not been generally adopted.

In this Letter, we analyze kinetic-energy spectra for fragment and light-charged-particle emission from target spectators following collisions of ${ }^{197} \mathrm{Au}+{ }^{197} \mathrm{Au}$ at $1000 \mathrm{MeV}$ per nucleon. We will show that the deduced slope temperatures and the much lower chemical breakup temperatures can be consistently understood if the intrinsic constituent motion of the decaying fermionic system is taken into account. With this interpretation, the slope temperatures reflect the bulk density of the system prior to its disintegration, and thus provide information on the mechanism of fragment formation.

Beams of ${ }^{197} \mathrm{Au}$ with incident energy $1000 \mathrm{MeV}$ per nucleon from the heavy-ion synchrotron SIS of the GSI Darmstadt were used to bombard ${ }^{197} \mathrm{Au}$ targets of $25-\mathrm{mg} / \mathrm{cm}^{2}$ areal thickness. As part of a larger experimental setup [10,27,28], three high-resolution telescopes were placed at backward angles for detecting the products 
of the target-spectator decay. Each telescope consisted of three Si detectors with thicknesses 50, 300, and $1000 \mu \mathrm{m}$ and of a 4-cm-long CsI(Tl) scintillator with photodiode readout and subtended a solid angle of $7.0 \mathrm{msr}$.

The products of the projectile decay were measured with the time-of-flight wall of the ALADIN spectrometer [9] and $Z_{\text {bound }}$ was determined event by event. The sorting variable $Z_{\text {bound }}$ is defined as the sum of the atomic numbers $Z_{i}$ of all projectile fragments with $Z_{i} \geq 2$. $Z_{\text {bound }}$ reflects the variation of the charge of the primary spectator system and is therefore correlated with the impact parameter of the reaction. Because of the symmetry of the collision system, the mean values of $Z_{\text {bound }}$ for the target and the projectile spectators within the same event class have been assumed to be identical. First results of these measurements have been presented in Ref. [10], including some of the kineticenergy data that will be discussed in the following.

Energy spectra of protons, measured at $\theta_{\mathrm{lab}}=150^{\circ}$ and sorted into eight bins of $Z_{\text {bound }}$, are shown in Fig. 1 (left panel). They are characterized by a hard component and an additional soft component that is most clearly identified at large $Z_{\text {bound. }}$. Fits using two Maxwellians yield temperature parameters of very different magnitude that both are nearly independent of $Z_{\text {bound }}$ (Fig. 1, right panels). The slope temperature $T$ of about 5 to $7 \mathrm{MeV}$ of the soft component is of the same order as the measured breakup temperatures [10]. Its intensity, at the same time, is strongly correlated with that of heavier fragmentation

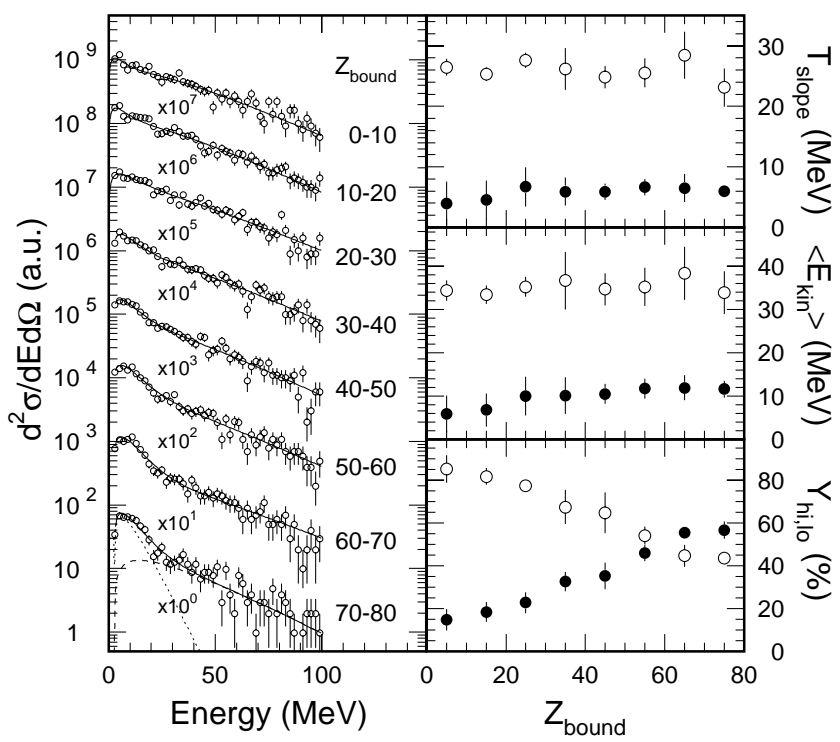

FIG. 1. Proton energy spectra measured at $\theta_{\text {lab }}=150^{\circ}$ (left panel) and slope temperatures, mean kinetic energies, and relative yields of the two components with high (open circles) and low (closed circles) temperatures (right panels). The full lines represent the fit results, and the dashed and dotted lines give the high- and low-temperature components for $70 \leq Z_{\text {bound }} \leq 80$ individually. Note that the fitting was performed with the assumption that two components exist which, for $Z_{\text {bound }} \leq 30$, is not unambiguously supported by the data. products [29] which suggests a, perhaps partial, interpretation as evaporation from highly excited residual nuclei. For the ${ }^{197} \mathrm{Au}+{ }^{12} \mathrm{C}$ reaction at the same energy, an equilibrium proton component with nearly identical properties was reported by Hauger et al. [22].

Two components have also been identified in the spectra of $\alpha$ particles (Fig. 2) and in the neutron data measured with LAND [30], with low-temperature components typical for evaporation. The high-temperature components of protons and neutrons seem to have their origin not only in the breakup stage but also in the earlier cascading stages of the collision; their slope parameters are rather large and, as established for the neutron case [30], vary with the bombarding energy.

The high-temperature component of $\alpha$ particles decreases from $T \approx 17 \mathrm{MeV}$ for small $Z_{\text {bound }}$ to $T \approx$ $13 \mathrm{MeV}$ for large $Z_{\text {bound }}$ (see below). Its mean value of $15 \mathrm{MeV}$ is close to the slope temperatures observed for other species with $A \geq 2$ which exhibit Maxwellian spectra to a good approximation (Fig. 2). Their slope temperatures were obtained from single-component fits with three-parameter functions that included a Coulomb potential $V_{c}$. The results for the interval $20 \leq Z_{\text {bound }} \leq 60$ are shown in Fig. 3. Apart from the protons with $T \approx 26 \mathrm{MeV}$, all spectra exhibit temperatures that are narrowly dispersed around a mean value of $17 \mathrm{MeV}$.

A mass-invariant temperature, at first sight, seems to provide direct evidence for equilibration of the kinetic degrees of freedom. On the other hand, Coulomb effects should contribute in proportion to the fragment charge, recoil effects may be important for heavier fragments, and the small but finite motion of the target spectator

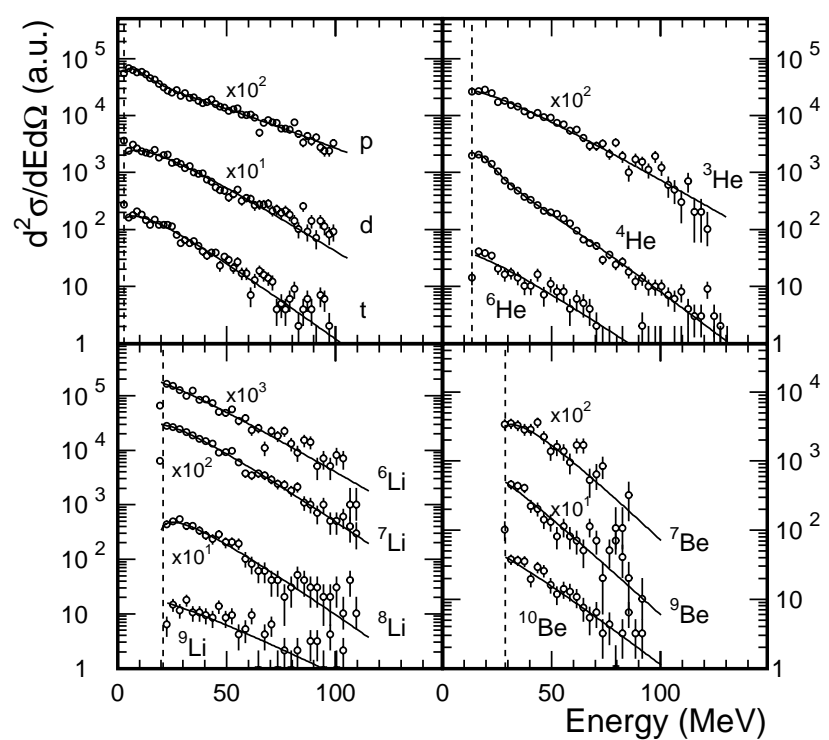

FIG. 2. Energy spectra of light charged particles and fragments with $Z \leq 4$ integrated over $20 \leq Z_{\text {bound }} \leq 60\left(\theta_{\text {lab }}=150^{\circ}\right)$. The full lines represent the fit results, and the dashed lines indicate the trigger threshold of the $300-\mu \mathrm{m}$ detector. The same normalization is used for all spectra. 


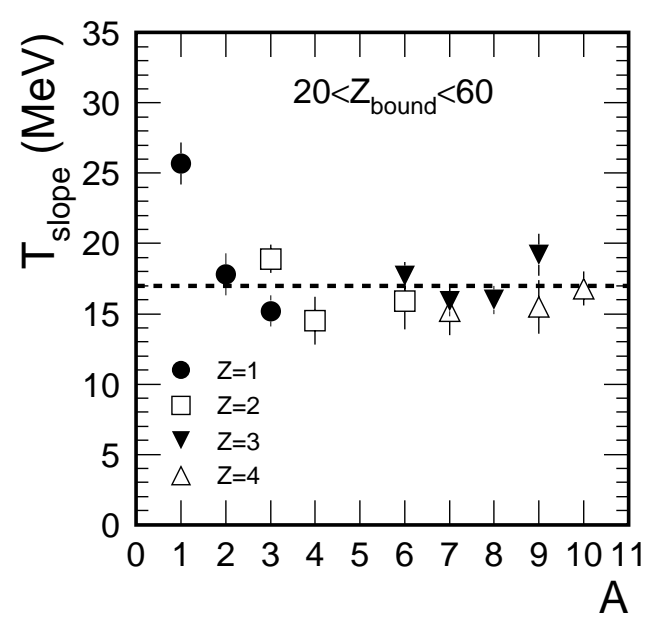

FIG. 3. Slope temperatures for isotopically resolved charged particles and fragments, measured at $\theta_{\mathrm{lab}}=150^{\circ}$ and integrated over $20 \leq Z_{\text {bound }} \leq 60$, as a function of the mass number $A$. For protons and $\alpha$ particles the value of the high-temperature component is shown.

should introduce a collective velocity component. In order to estimate the magnitude of these effects, a model study was performed which started from initial configurations generated by randomly placing fragments and light particles in a spherical volume with random momenta corresponding to a given temperature. Experimental charge distributions from Ref. [9] and an average density $\rho / \rho_{0}=$ 0.3 were used where $\rho_{0}$ is the normal nuclear density. $\mathrm{N}$-body Coulomb trajectory calculations were then performed and asymptotic slope temperatures were determined by fitting, following the same procedure as with the experimental data. The calculations showed that the considered effects are relatively small, perhaps of the order of $\Delta T / \Delta A \approx 0.2 \mathrm{MeV}$, and that they cancel each other to a good approximation at the backward angles chosen for the measurement. The measured slope parameters of $T \approx 17 \mathrm{MeV}$ (Fig. 3) are thus equal to the temperatures to be used in a thermal interpretation of the data.

It was suggested many years ago by Goldhaber that the product momenta in fast fragmentation processes may have their origin in the nucleonic Fermi motion within the colliding nuclei [25]. He also pointed out that the resulting behavior is indistinguishable from that of a thermalized system with rather high temperature. For the Fermi momentum $p_{F} \approx 265 \mathrm{MeV} / c$ of heavier nuclei the corresponding temperature is $T=15 \mathrm{MeV}$. This is intriguingly close to the measured slope temperatures $T=17 \mathrm{MeV}$.

Goldhaber's idea, initially formulated for cold nuclei, has been extended to the case of expanded fermionic systems at finite temperature by Bauer [19]. We have used the numerical solution reported there by assuming that the temperature of the system is given by the measured breakup temperatures $T_{\mathrm{HeLi}}$, derived from the yields of ${ }^{3,4} \mathrm{He}$ and ${ }^{6,7} \mathrm{Li}$ isotopes $[10,31,32]$. For the breakup density two alternative values $\rho / \rho_{0}=1.0$ and 0.3 were chosen which correspond to two significantly different scenarios for the process of fragment production. The higher value is expected for a fast abrasion stage that will induce fragmentation of the residual spectators but not instantly affect the nucleon motion within them. The lower value is in the range usually assumed for multifragment breakups of expanded systems $[1,2]$. Here the development of instabilities, as the system enters the spinodal region, will equally cause a rapid fragmentation, so that the nucleonic Fermi motion will contribute to the fragment kinetic energies. For a homogeneous system at a lower than normal density the Fermi motion is reduced and the effect will be smaller.

The slope temperatures obtained in this way are represented by the lines shown in Fig. 4. The comparison with the data is here restricted to the $A \leq 4$ isotopes for which the collected statistics are sufficient for studying the $Z_{\text {bound }}$ dependence. The recoil factor $\left(A_{s}-A\right) /\left(A_{s}-1\right)$ appearing in the Goldhaber formula $[19,25]$ can be safely ignored as the mass number $A_{s}$ of the spectator system is 100 to 150 on average and still about 50 in the bin of smallest $Z_{\text {bound }}$ [32]. Qualitatively, the predicted values are close to those observed. In particular, the rise with decreasing $Z_{\text {bound }}$ follows as a consequence of the rising breakup temperatures $T_{\mathrm{HeLi}}$. Apparently, with assumptions as made in the Goldhaber model, the two different types of temperatures are mutually consistent. The role of the slope temperatures, consequently, is restricted to describing the fragment motion while $T_{\mathrm{HeLi}}$ is the more suitable observable for representing the temperature of the nuclear environment at the breakup stage.

Recent calculations with transport models, which incorporate Fermi motion, support this interpretation [24,33].

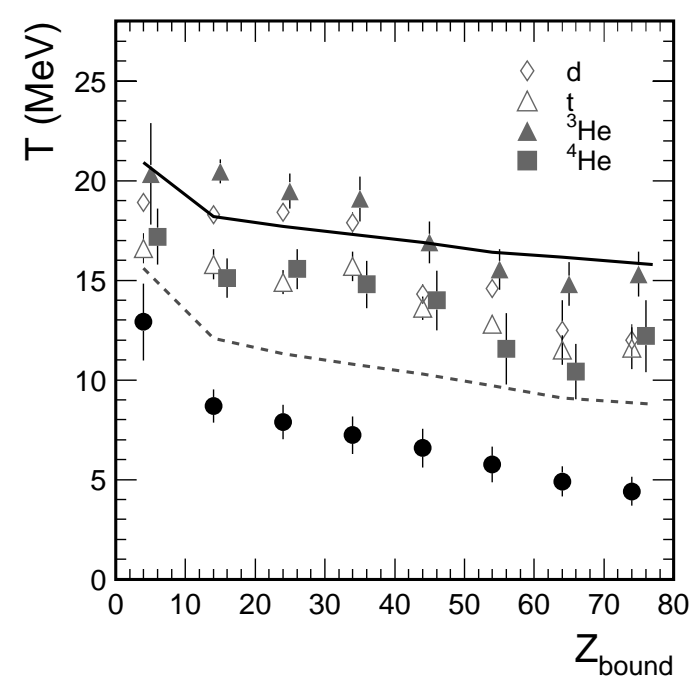

FIG. 4. Slope temperatures for light charged particles of mass $2 \leq A \leq 4$ (squares, triangles, and diamonds) and the isotope temperature $T_{\mathrm{HeLi}}$ (dots) as a function of $Z_{\text {bound }}$. The lines give the predictions for the fast breakup of a Fermi gas with finite temperature $T_{\mathrm{HeLi}}$ and with densities $\rho / \rho_{0}=1.0$ (full line) and 0.3 (dashed). 
The energies of spectator fragments, as measured in the present reaction, are well reproduced, and the coexistence of qualitatively different internal (or local) temperatures and fragment slope temperatures has been demonstrated in these quantum-molecular-dynamics (QMD) and Boltzmann-Nordheim-Vlasov (BNV) studies. On the experimental side, the observed similarity of fragment kinetic energy spectra, measured with different projectiles at various incident energies, is expected from the Goldhaber model $[9,15,23,26,34-36]$. The fluctuations corresponding to the higher slope temperatures may also explain the observed large widths of the intrinsic kinetic-energy distributions in ${ }^{197} \mathrm{Au}$ fragmentation at $600 \mathrm{MeV}$ per nucleon that are not easily reproduced with statistical multifragmentation models [37].

A more quantitative comparison with the data shown in Figs. 3 and 4 will favor the prediction for $\rho / \rho_{0}=1.0$ corresponding to a fast breakup over that for $\rho / \rho_{0}=0.3$ that is reached after a homogeneous expansion. This would not be unexpected in the present case of spectator fragmentation following collisions of heavy ions and might indicate a limited resilience of nuclear matter to shape deformations [38]. The cited QMD and BNV calculations consistently suggest that the fragments are preformed at an early stage of the collision $(\leq 50 \mathrm{fm} / c)$ before the system has expanded to typical breakup densities.

To draw firm conclusions at this time would certainly be premature. The role of secondary decays and possibly other effects would have to be considered, and a moderate collective velocity component as a result of thermal expansion cannot be completely excluded [37,39], even though it is not explicitly indicated for the present reaction (Fig. 3 and [9]). The main purpose of this work is to show that, within the Goldhaber picture of a random superposition of the nucleon momenta, the larger slope temperatures are fully consistent with a breakup at equilibrium temperatures of 6 to $8 \mathrm{MeV}$, as they are measured and also obtained with statistical multifragmentation models. Because of the sensitivity to the mode of fragment formation, via the density at which the Fermi motion has been established, this interpretation seems rather attractive and deserves further attention.

The authors thank J. Aichelin, C. Fuchs, T. Gaitanos, and H. H. Wolter for fruitful discussions. M. B., J.P., and C.S. acknowledge the financial support of the Deutsche Forschungsgemeinschaft under Contracts No. Be1634/1-1, No. Po256/2-1, and No. Schw510/2-1, respectively. This work was supported by the European Community under Contract No. ERBFMGECT950083.

*Present address: Fachbereich Physik, Bergische Universität, D-42119 Wuppertal, Germany.
†Present address: Department of Physics, Yale University, New Haven, CT 06512.

*Present address: Max-Planck-Institut für Kernphysik, D-69117 Heidelberg, Germany.

${ }^{\S}$ Present address: Max-Planck-Institut für Physik, D-80805 München, Germany.

[1] D. H. E. Gross, Rep. Prog. Phys. 53, 605 (1990).

[2] J. P. Bondorf et al., Phys. Rep. 257, 133 (1995).

[3] For a recent review of the field see, e.g., Proceedings of the International Workshop XXVII on Gross Properties of Nuclei and Nuclear Excitations, Hirschegg, Austria, edited by H. Feldmeier et al. (GSI, Darmstadt, 1999).

[4] A. S. Botvina and I. N. Mishustin, Phys. Lett. B 294, 23 (1992).

[5] H. W. Barz et al., Nucl. Phys. A561, 466 (1993).

[6] Bao-An Li et al., Phys. Lett. B 303, 225 (1993).

[7] J. Konopka et al., Prog. Part. Nucl. Phys. 30, 301 (1993).

[8] Yu-Ming Zheng et al., Phys. Rev. C 53, 1868 (1996).

[9] A. Schüttauf et al., Nucl. Phys. A607, 457 (1996).

[10] Hongfei Xi et al., Z. Phys. A 359, 397 (1997); Eur. Phys. J. A 1, 235 (1998).

[11] J. Lauret et al., Phys. Rev. C 57, R1051 (1998).

[12] S. P. Avdeyev et al., Eur. Phys. J. A 3, 75 (1998).

[13] Al. H. Raduta and Ad. R. Raduta, Phys. Rev. C 61, 034611 (2000).

[14] A. S. Botvina et al., Nucl. Phys. A584, 737 (1995).

[15] A. S. Hirsch et al., Phys. Rev. C 29, 508 (1984).

[16] J. Pochodzalla et al., Phys. Rev. C 35, 1695 (1987).

[17] D. J. Morrissey et al., Annu. Rev. Nucl. Part. Sci. 44, 65 (1994).

[18] For a review of the extensive work done in the intermediate-energy domain see, e.g., H. Fuchs and K. Möhring, Rep. Prog. Phys. 57, 231 (1994).

[19] W. Bauer, Phys. Rev. C 51, 803 (1995).

[20] K. B. Morley et al., Phys. Rev. C 54, 737 (1996).

[21] G. Wang et al., Phys. Rev. C 60, 014603 (1999).

[22] J. A. Hauger et al., Phys. Rev. C 57, 764 (1998).

[23] K. G. Gulamov et al., Phys. At. Nucl. 62, 533 (1999).

[24] P. B. Gossiaux et al., Nucl. Phys. A619, 379 (1997).

[25] A. S. Goldhaber, Phys. Lett. 53B, 306 (1974).

[26] For a review see J. Hüfner, Phys. Rep. 125, 129 (1985).

[27] V. Serfling et al., Phys. Rev. Lett. 80, 3928 (1998).

[28] S. Fritz et al., Phys. Lett. B 461, 315 (1999).

[29] T. Odeh, Ph.D. thesis, Universität Frankfurt, 1999.

[30] C. Gross, Ph.D. thesis, Universität Frankfurt, 1998.

[31] S. Albergo et al., Nuovo Cimento Soc. Ital. Fis. 89A, 1 (1985).

[32] J. Pochodzalla et al., Phys. Rev. Lett. 75, 1040 (1995).

[33] T. Gaitanos et al., Phys. Lett. B 478, 79 (2000).

[34] T. C. Sangster et al., Phys. Lett. B 188, 29 (1987).

[35] B. Berthier et al., Phys. Lett. B 193, 417 (1987).

[36] E. Renshaw Foxford et al., Phys. Rev. C 54, 749 (1996).

[37] M. Begemann-Blaich et al., Phys. Rev. C 58, 1639 (1998).

[38] M. Colonna et al., Phys. Rev. C 55, 1404 (1997).

[39] T. Lefort et al., e-print nucl-ex/9910017, 1999. 